\title{
A GaAs Pressure Sensor with Frequency Output based on Resonant Tunneling Diodes
}

\author{
K. Mutamba, M. Flath ${ }^{1}$, A. Sigurdardóttir and A. Vogt
}

\section{Introduction}

The work of the last two decades on RTDs has been dominated by the needs of semiconductor heterostructure devices for high frequency operation and high speed applications. The fast transit time of the quantum effect involved in the conduction mechanism and the exhibited negative differential resistance have motivated these extensive efforts. The development of the band-gap engineering has opened the possibility of using different material systems in order to extend RTD operation frequencies to the THz-region. Oscillations of up to $712 \mathrm{GHz}$ have been reported and several applications of RTDs for multivalued logic have been proposed [1-3]. However the RTD currentvoltage characteristics show, as explained below, a special uniaxial stress dependence, mainly in the negative differential resistance region. This dependence is used in this work to modulate the frequency of a relaxation oscillator which includes an $\mathrm{Al}_{0.6} \mathrm{Ga}_{0.4} \mathrm{As} / \mathrm{GaAs} \mathrm{RTD}$.

The suggested sensor matches almost all the requirements of modern sensors such as:

- the capability of monolithic integration with GaAsmicroelectronics

- the availability of a digital output

- an improved noise immunity of the output signal

Additional features of the sensor stem from the fact that:

\footnotetext{
${ }^{1}$ Now with SIEMENS AG, München
}

- the basic circuit practically consists only of a voltage source, an inductor and the RTD.

- the direct output is an instrinsic frequency modulated square wave without any additional converter circuit. This is important for measurements in a noisy environment.The square wave signal can be easily converted into an optical one if a LED is integrated in the immediate neighborhood of the RTD.

- the RTD can be integrated on different micromachined structures such as membranes, beams and other free standing structures used in integrated mechanical semiconductor sensors [5]. Such combinations offer a possibility of tailoring the sensor sensitivity for a wide range of pressures.

\section{Principal function of a RTD}

The RTD is a semiconductor device in which the conduction is determined by quantum-mechanical effects. The principal effects involved are the quantization of electron energies in a quantum well structure and the tunneling of electrons through thin barrier materials. In the RTD a compositional confinement is used to create wells and potentialbarrier structures of finite thickness and height. The $\mathrm{Al}_{x} \mathrm{Ga}_{1-\mathrm{x}} \mathrm{As} / \mathrm{GaAs} \mathrm{RTD}$ consists of a so-called double barrier heterostructure comprising a GaAs quantum well and two AlGaAs potential barrier structures. The undoped double barrier structure is sandwiched between two n-doped GaAs contact regions which play the role of electron suppliers. Due to the confinement only nearly discrete energy levels are allowed for electrons in the well. Applying a voltage to the RTD 
lets the current flow between the two outside electrodes and resonant tunneling occurs when the energy of the incoming electrons coincides with the energy levels created in the well. This resonant enhancement of the conductivity is a very fast process and gives rise to a differential negative resistance in the current-voltage characteristics [4]. These two features and the potential they offer for high frequency applications have motivated the whole amount of work on RTDs.

\section{The Effect of Pressure}

It has been demonstrated that the change of the position of the energy states in the quantum well with applied external pressure can be attributted to two major effects : the pressure-induced change in the electrons effective mass and the generated piezoelectric fields inside the well and the barrier materials. Hydrostatic pressure induces a variation of carrier effective mass which shifts the energy states in the well [6]. In the case of uniaxial and biaxial pressures, the piezoelectric field induced contribution to the energy shift is stronger than the one related to the effective mass change [7].

The variation in the position of the energy states through pressure modifies the current-voltage characteristics of the RTD. The observed effects are principally a shift of the peak and the valley voltages and a shift of the peak and the valley currents [8]. Both effects have an influence on the relaxation oscillator frequency.

\section{The Relaxation Oscillator}

A relaxation oscillator produces a square wave like waveform. The Oscillator consists of a voltage supply, a serial inductor, a RTD and a capacitor (Fig. 1) [9]. The oscillations are due to the DC instabilities caused by the RTD when biased in the negative differential region (NDR). The frequency is mainly determined by the serial inductance value, the shape of the RTD current-voltage characteristic and the bias voltage.

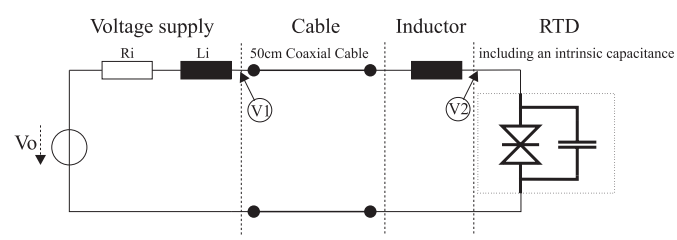

Fig. 1. The RTD circuit including the coaxial cable of the measurement setup
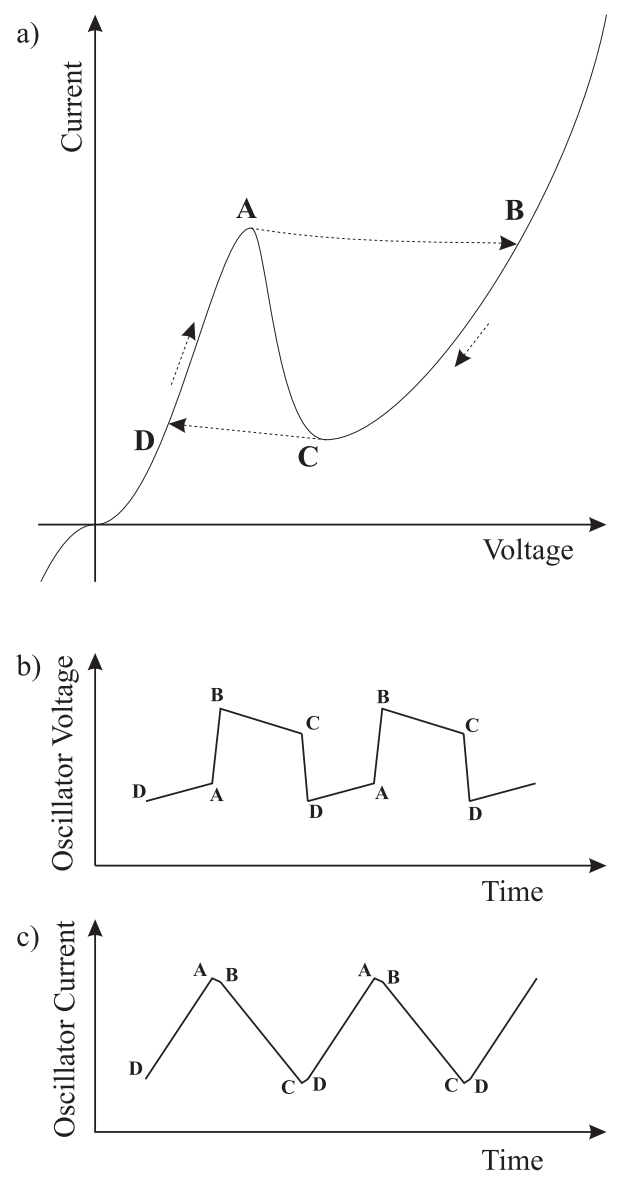

Fig. 2. a) RTD-I-V characteristics. The device is biased in the NDR region and the arrows indicate the I$\mathrm{V}$ excursion of the RTD during an oscillation cycle including the important points of the curve. b) and c) simplified oscillator voltage and current waveforms.

For moderate currents and frequencies, intrinsic parasitic capacitances can be used instead of an external one. The capacitance determines the rising and the falling times of the oscillator voltage edges (Fig. 2b). A square wave form can be assumed for the oscillator voltage. Therefore the influence of the capacitance on the frequency is minor as compared to the inductance.

When the RTD is biased in the NDR region the principal function of the relaxation oscillator can be explained as following (Fig. 2a,b and c) : 
- First, the current rises till it reaches the peak value (Point A).

- The serial inductance, the parallel capacitor and the NDR force the diode to switch from A to B. The inductance maintains the current while the capacitor takes up the difference current and therefore increases the RTD voltage. A fast switching from A to B occurs.

- The voltage reached is above the bias point and can not be maintained by the voltage source. Therefore the voltage decrease with a time constant determined through the inductor and the nonlinear RTD positive resistance.

- If the valley voltage (Point C) is reached a fast switch to point $\mathrm{D}$ takes place. The diode current increases while the inductor current remains constant. The current difference is supplied by the capacitor.

\section{Circuit analysis}

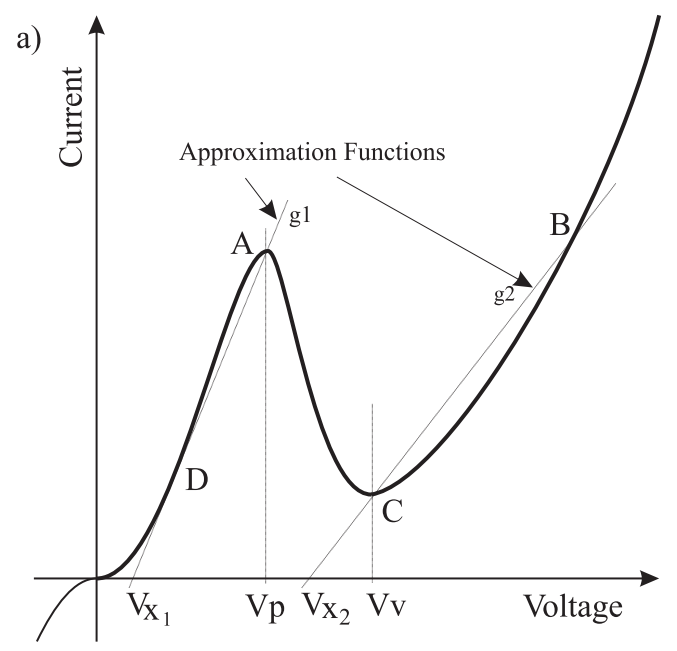

b)

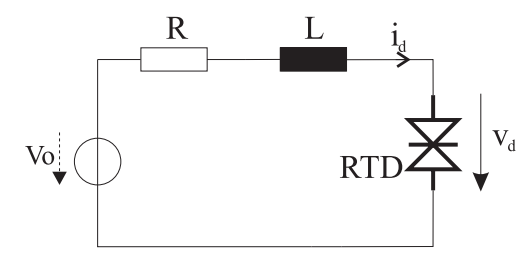

Fig. 3. a) Linear approximation of the positive conductance regions. b) Schematic of the analyzed circuit.

To study the influence of the circuit elements and the IV curve form on the oscillation frequency, we propose a systematic circuit analysis, by solving the circuit differential equation. For an analytical solution some approximations are necessary. The circuit to be analysed is drawn in Fig. 3b. Obviously the capacitor can be neglected. It determines mainly the switching times from $\mathrm{A}$ to $\mathrm{B}$ and D to A (Fig. 2b). These transitions are very sharp and the corresponding switching times are very short when compared to the times from $\mathrm{B}$ to $\mathrm{C}$ and $\mathrm{C}$ to $\mathrm{D}$ which determine the two alternances of the waveform. Therefore we only have to calculate the time needed for the D to A and B to C transitions respectively.

From the schematic of Fig. 3b we can write:

$$
V_{o}=R i_{d}+L \frac{d v_{d}}{d t}+v_{d}
$$

$$
i_{d}=g\left(v_{d}\right)
$$

and

$$
h=\frac{d g}{d v_{d}}
$$

Eqn. (1) can be written as

$$
V_{o}=(g R+1) v_{d}+g L \frac{d v_{d}}{d t}+h L v_{d} \frac{d v_{d}}{d t}
$$

The next simplification will be to approximate the I$\mathrm{V}$ curve between the transistions $\mathrm{D} \rightarrow \mathrm{A}$ and $\mathrm{B} \rightarrow \mathrm{C}$ through linear functions, so that $\mathrm{g}$ is constant in the two positive resistance regions respectively. Details are demonstrated in Fig. 3a. The equation can then be easily solved by using the following assumption :

$$
i_{d}=g v_{d}+i^{*}
$$

where

$$
i^{*}=-g V_{x}
$$

For solving the differential equation a boundary condition is necessary. If the transition from point $\mathrm{D}$ to $\mathrm{A}$ ( $\mathrm{B}$ to $\mathrm{C}$ ) is set to begin at $\mathrm{t}=0$ and the corresponding voltage at point $\mathrm{D}(\mathrm{B})$ is $V_{D}\left(V_{B}\right)$ 
the solution for the entire oscillation period, under the assumption that $g R$ is much less than 1 , is

$$
\begin{gathered}
t=g_{1} L\left[\ln \left(\frac{V_{D}-R g_{1} V_{x I}-V_{0}}{V_{p}-R g_{1} V_{x I}-V_{o}}\right)\right]+ \\
g_{2} L\left[\ln \left(\frac{V_{B}-R g_{2} V_{x 2}-V_{0}}{V_{v}-R g_{2} V_{x 2}-V_{0}}\right)\right]
\end{gathered}
$$

Looking at this equation, it can be stated that the oscillation frequency is inversely proportional to $L$.

Two effects of almost the same importance are observed when pressure is applied on the RTD. The peak and the valley voltages change as well as the peak and the valley currents. We can separate these effects in order to study their influences on the oscillation frequency and their respective contributions to the pressure sensitivity of the sensor. For a defined bias voltage, shifting only the peak and the valley voltages can be compared to the effect of changing the position of the bias point in the NDR. Using Eqn.(5) a maximum oscillation frequency is reached when $V_{0}$ varies from $\quad V_{p}-R g_{1} V_{x 1}$ to $V_{v}-R g_{2} V_{x 2}$. Assuming that $R g_{i} V_{x i}$ is small compared to $V_{p}$ and $V_{v}$ one can speak about $V_{0}$ varying between $V_{p}$ and $V_{v}$. The oscillation frequency decreases to zero for $V_{O}$ approaching the border of the NDR (nearly $\left.V_{p, v}\right)$. The peak and the valley voltages shift, for example, to smaller values with applied compressive pressures. The corresponding maximum of the bias dependent frequency curve occurs at a smaller value of $V_{0}$. The oscillation frequencies are then increased in the bias region before the maximal frequency and decreased in the region above. This leads to the first observation that the minimal pressure sensitivity is obtained with the diode biased for the maximal frequency. But moving the bias voltage toward the border of the NDR gives rise to a growing sensitivity. The highest one is reached for $V_{0}$ close to $V_{p, v}$, but it is useful to assure that the circuit does not get out of oscillations. This is important in order to achieve a good compromise between stable oscillations and high sensitivity.

The influence of the current shifts can be estimated as following: for a fixed value of $L, V_{D}$ and $V_{B}$ are determined by the peak and the valley currents respectively. A relation of proportionality can be assumed in a first approximation. The peak and valley current variation leads obviously to respective changes of $g_{1}, V_{x 1}$ and $g_{2}, V_{x 2}$ of the oscillation frequency equation. In the case of a compressive pressure the peak and the valley current shift both to smaller values. From our measurements, the change in the peak current is higher $(\sim 10 \%)$ than for the valley current $(\sim 6 \%)$ in the pressure range used. It can then be inferred from Eqn. (5) that the current change affects the two terms differently. Eqn. (5) also shows that increasing the current will lower the first term while increasing the second. The other parameters will then determine whether the oscillation frequency will be decreased or increased.

Another important conclusion follows from the above descriptions:

The bias point is shifted in the same direction indepedent of the bias voltage value in the NDR region. The existence of a maximum in the oscillation frequency gives rise to two interesting operation regions. A growing bias dependent sensitivity is obtained in the two regions. The peak and valley current effects have shown to shift the oscillation frequency. The useful biasing region will be where the combination of the two effects increases the frequency shift leading to an higher pressure sensitivity. This assumes the existence of a bias regions where the effects compensate partially each other and another one of mutual reenforcement.

\section{Experimental Results and Discussion}

The used RTD-sensors for frequency measurements have been fabricated by MBE (Molecular Beam Epitaxy) on a (001)-oriented GaAs substrate. Rectangular sensors have been obtained from the wafer. The oscillator circuit including RTD-biasing circuit, external serial inductance and the connecting coaxial cable is presented in Fig. 1.

The applied bias voltage is $V_{1}$ and the measured oscillator voltage is $V_{2}$. The bias was supplied by a precision voltage supply with $L_{i}=3 \mu H$ and a $R_{i}$ value of $5 \mathrm{~m} \Omega$. An external inductance $L=30 \mu \mathrm{H}$

was used. The following room temperature parameters have been measured for the $\mathrm{Al}_{0.6} \mathrm{Ga}_{0.4} \mathrm{As} / \mathrm{GaAs} \mathrm{RTDs}$ without applied uniaxial pressure: a current Peak to valley ratio (PVR) of 1.44, peak and valley voltages of 0.74 and $0.87 \mathrm{~V}$ respectively and the corresponding peak and valley currents of 55.5 and $38.5 \mathrm{~mA}$. A PSPICE simulation of the entire circuit was carried out using a modified version of the RTD-model proposed by Brown et al. [10] with an exponential form of the excess current. The range of the measured pressure dependent oscillation frequencies was confirmed by the calculations. Uniaxial compressive pressures have 
been applied in the [110]-direction using a measurement setup with a lever system. Forces of defined magnitude could be applied on two sensor edges.The pressure dependent frequency measurements and the deduced absolute pressure sensitivities are presented in Figs. 4. The pressure effects appear as qualitatively discussed.

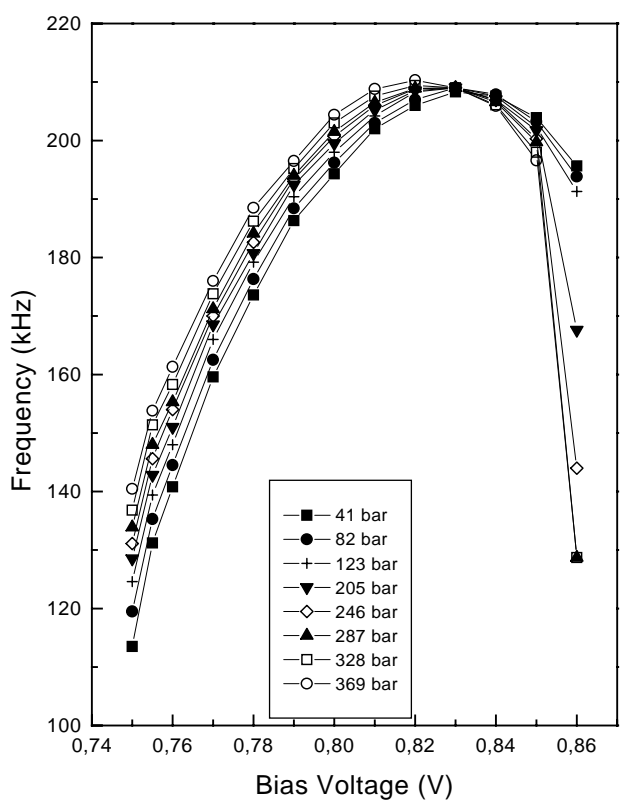

Fig. 4. Room temperature pressure dependent frequency measurement. There is a minimal sensitivity region.

Two regions of growing sensitivities as well as a minimum sensitivity are observed. The sensitivity curve shows a possibility of improvement up to 80 $\mathrm{kHz} / \mathrm{kbar}$ at $113 \mathrm{kHz}$ with stable oscillations. But as mentioned before, bias points closer to the peak or valley voltages have to be avoided. The minimal and the maximal bias voltages at which the diode can oscillate are shifted to smaller values when the applied pressure increases.

According to this fact the bias region below the maximal frequency of the zero pressure curve can be used with a relative high sensitivity and reduced risk of switching the sensor out of oscillations. The exact value of the initial bias voltage can then be determined by the range of pressures to be measured and the corresponding full scale shift of the RTD's currentvoltage curves. However the effect of temperature on the oscillation frequency and the sensor sensitivity has to be studied. In fact, increasing temperature is known, in a first approximation, to increase the thermionic current above the barriers of the RTD. This results in an increase of the valley current which then reduce the PVR and affects the oscillation frequency [11]. A temperature compensation can be achieved by a temperature dependent control of the bias point position. The range of uniaxial pressures used in this work can be easily reached by integrating the RTD on an appropriate micromechanical structure such as a selectively etched AlGaAs or GaAs membrane for differential pressure measurements, or a free standing beam for acceleration sensors.

\section{Conclusion}

A RTD based bulk pressure sensor with a frequency output has been presented. A simple analytic description of the relaxation oscillator circuit has permitted to extract useful fundamental relations for the oscillator design as well as the sensor sensitivity optimization. Different measurement ranges and sensitivities can be defined by changing simply the bias point of the RTD in the negative resistance region.

\section{References}

[1] E.R. Brown, J. R. Söderström, C. D. Parker, L. J. Mahoney, K. M. Molvar and T. C. Mc Gill, " Oscillations up to $712 \mathrm{GHz}$ in InAs/AlSb resonant tunneling diodes at room temperature ", Appl. Phys. Lett., vol 58, p. 2291, 1991.

[2] J. Söderström and T. G. Anderson, " A multiplestate memory cell based on resonant tunneling structures for signal processing in three-state logic ", IEEE Electron Device Lett., vol. 9, pp. 2163-2164, 1988.

[3] Z. X. Yan and M. J. Deen, " A new resonantTunnel Diode-based Multivalued Memory circuit using a MESFET depletion load "; IEEE J. of solid-state circuits, vol. 27, no. 8, pp. 11981202, Aug. 1992.

[4] J. F. Whitaker, G. A. Mourou, T. C. L. G. Sollner and W. D. Goodhue ", Picosecond switching time measurement of a resonant tunneling diode ", Appl. Phys. Lett., vol. 53, no. 5, pp. 385-387, Aug. 1988.

[5] K. Fobelets, R. Vounckx, and G. Borghs, " A GaAs pressure sensor based on resonant tunneling diodes ", J. Micromech. Microeng. 4, pp. 123-128, 1994.

[6] A. Di Carlo and P. Lugli, " Valley mixing in resonant tunneling diodes with applied hydrostatic pressure ", Semicond. Sci. Technol., vol. 10, pp. 1673-1679, 1995.

[7] J. D. Albrecht, L. Cong, P. P. Ruden, M. I. Nathan and D. L. Smith, " Resonant tunneling in 
(001)- and (111)-oriented III-V double barrier heterostructures under transverse and longitudinal stresses ", J. Appl. Phys., vol. 79, no. 10, pp. 7763-7769, May 1996.

[8] K. Mutamba, A. Vogt, A. Sigurdardóttir, M. Flath, J. Miao, A. Dehé, I. Aller and H. L. Hartnagel, " Uniaxial stress dependence of $\mathrm{AlGaAs} / \mathrm{GaAs}$ RTD characteristics for sensor applications ", Proc. MME'96 conference, Barcelona, Spain, pp. 85-89, 1996.

[9] Woo F. Chow, " Principles of tunnel diode circuits ", J. Wiley and Sons, Inc., 1964.

[10] E. R. Brown, O. B. McMahon, L. J. Mahoney and K. M. Molvar, " SPICE model of the resonant tunneling diode ", Electr. Lett., vol. 32, no. 10, May 1996.

[11] O. Vanbésien, R. Bouregba, P. Mounaix and D. Lippens, " Temperature dependence of the peak to valley current ratio in resonant tunneling double barriers ", Resonant tunneling in semiconductors, Physics and applications, NATO ASI Series, Serie B, Physics vol. 277, pp. 107-116, Plenum Press, 1991. 